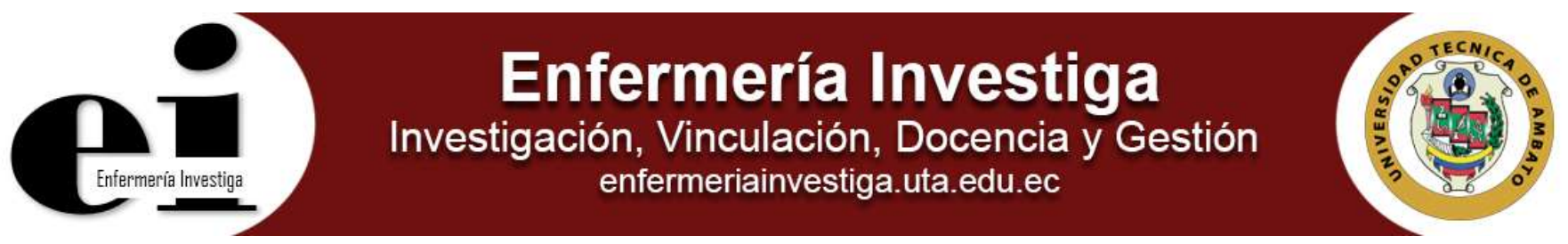

Artículo original

\title{
Escala de Alvarado como herramienta diagnóstica para apendicitis aguda
}

Alvarado Score as a diagnostic tool for acute apendicitis

Iván Pimienta Concepción ${ }^{1}$, María Isabel Fong Betancourt¹, Raúl González Salas ${ }^{1}$, Liset Camaño Carballo¹, Tatiana Carola Serrano Serrano¹, Graciela de las Mercedes Quishpe Jara², Verónica del Pilar Gavilanes Fray²

\footnotetext{
${ }^{1}$ Carrera de Medicina, Facultad de Ciencias Médicas, Universidad Regional Autónoma de los Andes, Ambato, Ecuador.

${ }^{2}$ Carrera de Enfermería, Facultad Ciencias de la Salud, Universidad Técnica de Ambato, Ambato, Ecuador.
}

Pimienta Cl, et al. Escala de Alvarado como herramienta diagnóstica para apendicitis aguda. Enferm Inv (Ambato). 2017; 2(2):68-71.

2477-9172 / 2550-6692 Derechos Reservados (C 2017 Universidad Técnica de Ambato, Carrera de Enfermería. Este es un artículo de acceso abierto distribuido bajo los términos de la Licencia Creative Commons, que permite uso ilimitado, distribución y reproducción en cualquier medio, siempre que la obra original es debidamente citada.

\section{Historia:}

Recibido: 08 mayo 2017

Revisado: 10 mayo 2017

Aceptado: 11 mayo 2017

Palabras Claves: Escala de Alvarado; dolor abdominal; apendicitis; apendicectomía

Keywords: Alvarado Score; abdominal pain; appendicitis appendectomy

\begin{abstract}
Resumen
Introducción: La apendicitis aguda es la enfermedad intrabdominal más frecuente tratada de urgencia. Resulta de interés la utilización de la Escala de Alvarado en el diagnóstico de esta patología por su contribución a la disminución de apendicectomías negativas.

Objetivo: Determinar la validez de la Escala de Alvarado como herramienta diagnóstica para apendicitis aguda en pacientes atendidos en el Servicio de Cirugía General del Hospital IESS Ambato.

Métodos: Se realizó una investigación observacional, descriptiva y transversal en pacientes hospitalizados con cuadro de dolor abdominal y sospecha de apendicitis aguda, valorados en el Servicio de Cirugía General en el Hospital General IESS Ambato en el período junio 2015 a noviembre del año 2015.

Resultados: De acuerdo al resultado histopatológico predominó la apendicitis aguda supurada con $(n=83,54.9 \%)$; seguida de apendicitis aguda gangrenosa ( $n=35,23.2 \%)$; apendicitis aguda precoz $(n=15,9.9 \%)$; mientras que el resultado normal y apendicitis aguda gangrenosa perforada se encontraron con los mismos valores $(n=9,5.9 \%)$. Conclusiones: Existió un puntaje elevado de la Escala de Alvarado para el diagnóstico de apendicitis aguda según la severidad de los hallazgos descritos en el resultado histopatológico posterior a la apendicectomía. El resultado histopatológico de mayor frecuencia fue la apendicitis aguda supurada, con un riesgo medio en la Escala de Alvarado, la cual es más sensible en periodos de gravedad.
\end{abstract}

Introduction: Acute appendicitis is the most common intra-abdominal disease treated urgently. It is of interest the use of the Alvarado Score in the diagnosis of this pathology for its contribution to the decrease of negative appendectomies.

Objetive: To determine the validity of the Alvarado Score as a diagnostic tool for acute appendicitis in patients treated at the General Surgery Service of the IESS Ambato Hospital.

Methods: An observational, descriptive and crosssectional investigation was carried out in hospitalized patients with abdominal pain and suspicion of acute appendicitis, evaluated at the General Surgery Service at the IESS Ambato General Hospital from june 2015 to november 2015. Results: According to the histopathological result, acute appendicitis was suppurated with $(n=83$, $54.9 \%$ ); Followed by acute gangrenous appendicitis $(n=35,23.2 \%)$; Acute appendicitis $(n=15,9.9 \%)$; While normal and acute perforated gangrenous appendicitis were found with the same values $(n=9$, $5.9 \%)$.

Conclusions: There was a high score of the Alvarado Score for the diagnosis of acute appendicitis according to the severity of the findings described in the histopathological result after the appendectomy. The most frequent histopathological result was acute suppurative appendicitis, with an average risk in the Alvarado Score, which is more sensitive in periods of severity. 


\section{Introducción}

La primera apendicetomía reportada se practicó en 1735 y la descripción histopatológica de la inflamación del apéndice con posterior evolución hacia peritonitis fue publicada por Fitz en $1886^{1}$.

La apendicitis aguda es la enfermedad intrabdominal más frecuente tratada de urgencia que puede llevar a la muerte de no recibir asistencia médica de forma correcta. Según documentos históricos Hipócrates conocía la existencia de procesos agudos dentro del abdomen; por lo que durante años el dolor en la fosa iliaca derecha se consideró que era causa de tiflitis y las consecuencias graves eran producto de la misma².

El apéndice vermiforme es un pequeño segmento de intestino que se localiza en la unión del intestino delgado con el grueso, en el cuadrante inferior derecho del abdomen. Pese a que en niños se le atribuye cierto grado de función en la inmunidad, en adultos carece de función o utilidad $^{3}$.

En la actualidad la apendicitis aguda conlleva a una cirugía de urgencia con elevada incidencia, siempre y cuando el diagnóstico se haga de forma oportuna. Este diagnóstico contempla pruebas de imagen como la ecografía y la tomografía axial computarizada (TAC), las que tienen una sensibilidad cercana al $100 \%{ }^{4}$. No obstante algunos autores se basan únicamente en el criterio clínico, sus conocimientos y experiencias. Un diagnóstico precoz evita complicaciones como la perforación y la peritonitis aguda, poniendo en riesgo la vida del paciente ${ }^{5}$.

Se realizan esfuerzos para reducir el número de cirugías que se practican innecesariamente, lo que ha contribuido a reducir la morbilidad y la mortalidad, así como las complicaciones asociadas a esta práctica quirúrgica. Sin embargo, el proceso diagnóstico constituye un desafío de gran magnitud y exige la pericia de los cirujanos, teniendo en cuenta que la automedicación previa de los pacientes y la tardanza en procesos administrativos provoca mayor incidencia de complicaciones y el incremento de la estadía hospitalaria 6 .

En 1986 el doctor Alfredo Alvarado planteó la utilidad de establecer criterios de clasificación sencilla y confiable que permitan aproximar en forma oportuna el diagnóstico de apendicitis aguda. Para ello desarrolló una escala diagnóstica de apendicitis aguda, basada en la calificación de tres síntomas, tres signos clínicos y dos valores de hemograma ${ }^{7}$.

Se denomina apendicectomía negativa cuando el reporte de patología del apéndice resecado es normal, pudiendo encontrarse un apéndice normal, pero con otra patología intraabdominal, mientras que en la apendicectomía innecesaria puede encontrarse el apéndice normal sin ninguna patología evidente. La apendicectomía negativa habitualmente es llevada a cabo ante la duda diagnóstica, o bien con la intención del cirujano de evitar una perforación apendicular y sus complicaciones subsecuentes ${ }^{8,9}$.

El objetivo de la presente investigación fue determinar la validez de la Escala de Alvarado como herramienta diagnóstica para apendicitis aguda en pacientes atendidos en el Servicio de Cirugía General del Hospital IESS Ambato.

\section{Materiales y Métodos}

Se realizó una investigación observacional, descriptiva y transversal en pacientes hospitalizados con cuadro de dolor abdominal y sospecha de apendicitis aguda, valorados en el Servicio de Cirugía General en el Hospital IESS Ambato durante el período de junio 2015 a noviembre del año 2015. El universo de estudios estuvo constituido por 151 pacientes, no se seleccionó muestra. La información se recolectó a partir de las historias clínicas de los pacientes como fuentes de información secundaria. La Escala de Alvarado fue aplicada en el preoperatorio y se relacionó con el reporte histopatológico. Para el procesamiento de los datos se utilizó el paquete estadístico SPSS versión 22 trial IR versión libre.

Se incluyeron las historias clínicas de los pacientes que ingresaron al Servicio de Emergencia de Cirugía General con cuadro probable de apendicitis aguda; con edades entre los 18 y 65 años y que cumplieron con todos los parámetros de la Escala de Alvarado teniendo en cuenta la probabilidad de apendicitis aguda como se describe a continuación:

- Riesgo bajo: 0 a 4 puntos,

- Riesgo medio: 5 a 7 puntos,

- Riesgo alto: 8 a 10 puntos.

Se excluyeron todas las historias clínicas de pacientes menores de 18 años y sin diagnóstico probable de apendicitis aguda por no cumplir con los parámetros de la Escala de Alvarado, pacientes que no fueron sometidos a apendicetomía o laparotomía exploratoria por negativa a aceptar el tratamiento quirúrgico o por falta de consentimiento informado; así como aquellos en los que no fue posible la recolección de los parámetros de la Escala de Alvarado o por falta de reporte del apéndice extirpado.

Se respetaron los principios de la Ética Médica basado en lo establecido en la Declaración de Helsinki para la realización de estudios investigativos en seres humanos.

\section{Resultados}

Del total de pacientes diagnosticados con apendicitis aguda en el periodo de junio a noviembre del año 2015, la mayoría estuvieron comprendidos entre 26 y 35 años de edad ( $n=65$, $43.1 \%$ ) y hubo predominio del género masculino ( $n=97$, $64.2 \%$ ) (tabla 1).

Predominó el riesgo medio según la valoración de la Escala de Alvarado $(n=73,48.3 \%)$, con predomino del sexo masculino $(n=50,33.1 \%)$. De igual forma el sexo masculino $(n=29,19.2 \%)$ predominó en los pacientes valorados de riesgo alto y en los pacientes valorados de riesgo bajo $(\mathrm{n}=18,11.9 \%)$ (tabla 2).

El análisis del resultado histopatológico permitió constatar que un mayor número de pacientes $(n=83,54.9 \%)$ presentó apendicitis aguda supurada. A continuación, se ubicaron los pacientes con apendicitis aguda gangrenosa $(n=35,23.2 \%)$ y apendicitis aguda precoz $(n=15,9.9 \%)$; por último, la apendicitis aguda gangrenosa perforada y la apendicitis aguda normal $(n=9,5.9 \%)$ (tabla 3$)$. 
Tabla 1. Frecuencia de apendicitis aguda según edad y sexo, Hospital IESS Ambato, junio a noviembre del año 2015.

\begin{tabular}{lccc}
\hline $\begin{array}{l}\text { Edad } \\
\text { (años)/Sexo }\end{array}$ & $\begin{array}{c}\text { Sexo masculino } \\
\mathbf{n}(\%)\end{array}$ & $\begin{array}{c}\text { Sexo femenino } \\
\mathbf{n}(\%)\end{array}$ & $\begin{array}{c}\text { Total } \\
\mathbf{n}(\%)\end{array}$ \\
\hline$<25$ & $19(12.6)$ & $11(7.3)$ & $30(19.9)$ \\
$\geq 26$ y $\leq 35$ & $44(29.1)$ & $21(13.9)$ & $65(43.1)$ \\
$\geq 36$ y $\leq 45$ & $17(11.3)$ & $15(9.9)$ & $32(21.2)$ \\
$\geq 46$ y $\leq 55$ & $14(9.3)$ & $5(3.3)$ & $19(12.6)$ \\
$\geq 56$ y $\leq 65$ & $3(1.9)$ & $2(1.3)$ & $5(3.3)$ \\
\hline Total & $\mathbf{9 7 ( 6 4 . 2 )}$ & $\mathbf{5 4 ( 3 5 . 8 )}$ & $\mathbf{1 5 1 ( 1 0 0 )}$ \\
\hline Fuente: Historias clínicas Hospital IESS Ambato. & & \\
\hline
\end{tabular}

Tabla 2. Valoración de la Escala de Alvarado según sexo, Hospital IESS Ambato, junio a noviembre del año 2015.

\begin{tabular}{lccc}
\hline Valoración/Sexo & $\begin{array}{c}\text { Sexo masculino } \\
\mathbf{n}(\%)\end{array}$ & $\begin{array}{c}\text { Sexo femenino } \\
\mathbf{n}(\%)\end{array}$ & $\begin{array}{c}\text { Total } \\
\mathbf{n}(\%)\end{array}$ \\
\hline Riesgo bajo & $18(11.9)$ & $12(7.9)$ & $30(19.9)$ \\
Riesgo medio & $50(33.1)$ & $23(15.2)$ & $73(48.3)$ \\
Riesgo alto & $29(19.2)$ & $19(12.6)$ & $48(31.8)$ \\
\hline Total & $\mathbf{9 7 ( 6 4 . 2 )}$ & $\mathbf{5 4 ( 3 5 . 8 )}$ & $\mathbf{1 5 1 ( 1 0 0 )}$ \\
\hline
\end{tabular}

Fuente: Historias clínicas Hospital IESS Ambato.

Tabla 3. Diagnóstico histopatológico de apendicitis aguda, Hospital IESS Ambato, junio a noviembre del año 2015.

\begin{tabular}{lcccc}
\hline Diagnóstico & $\mathbf{n}$ & \% & \% Válido & \% Acumulado \\
\hline Normal & 9 & 5.9 & 5.9 & 6 \\
Precoz & 15 & 9.9 & 9.9 & 15.9 \\
Supurada & 83 & 54.9 & 55 & 70.9 \\
Gangrenosa & 35 & 23.2 & 23.2 & 94 \\
Gangrenosa perforada & 9 & 5.9 & 5.9 & 100 \\
\hline Total & $\mathbf{1 5 1}$ & $\mathbf{1 0 0}$ & $\mathbf{1 0 0}$ & \\
\hline Fuente: Historias clínicas Hospital IESS Ambato & & & &
\end{tabular}

\section{Discusión}

Se realizó una comparación con el estudio realizado en el Centro Médico ABC Campus Santa $\mathrm{Fe}$, en el que se clasificaron los pacientes según la Escala de Alvarado en riesgo bajo (0-3 puntos), intermedio (4-6 puntos) o alto ( $>7$ puntos) de urgencia abdominal médica o quirúrgica. Alcanzando resultados del $39 \%$ para riesgo bajo, $44 \%$ riesgo intermedio y $16 \%$ riesgo alto, los resultados fueron semejantes a la presente investigación ${ }^{10-12}$.

Al revisar otro estudio realizado en el Hospital Clínico Quirúrgico Freyre de Andrade en el período de un año, donde se seleccionaron pacientes con diagnóstico inicial de apendicitis aguda a quienes se les aplicó la Escala de Alvarado y se comprobó la enfermedad histológicamente en un $86 \%$ de los pacientes operados, donde solo siete pacientes $(14 \%)$ presentaron apéndice cecal normal ${ }^{13-15}$.

En la presente investigación los pacientes incluidos en el grupo de alto riesgo (8-10 puntos) de la escala de Alvarado, se les confirmó de igual manera el diagnóstico de apendicitis aguda en relación con la histología, corroborando la veracidad de la escala al compararla con otros estudios ${ }^{16}$.

Al existir la concordancia de la escala con el resultado histopatológico, se mostró en este estudio que la apendicitis aguda negativa o apéndices normal tan solo fue del $5.9 \%$, representando cifras bajas en relación con el Hospital Clínico Quirúrgico Freyre de Andrade. En una mayor proporción se encontraron los pacientes de riesgo medio en correspondencia con el resultado histopatológico de apendicitis aguda supurada ${ }^{13,15,17}$.

En referencia a los días de hospitalización, los pacientes que fueron apendicectomizados permanecieron de uno a dos días en la institución hospitalaria. En un estudio realizado en la Universidad del Azuay se obtuvo un tiempo promedio de estancia hospitalaria por apendicectomía abierta de 4,1 días, en cambio el tiempo fue de 2,7 días en pacientes con apendicectomía laparoscópica ${ }^{18,19}$.

\section{Conclusiones}

Existió un puntaje elevado de la Escala de Alvarado para el diagnóstico de apendicitis aguda según la severidad de los hallazgos descritos en el resultado histopatológico posterior a la apendicectomía. El resultado histopatológico de mayor frecuencia fue la apendicitis aguda supurada, con un riesgo medio en la Escala de Alvarado, la cual es más sensible en periodos de gravedad.

\section{Referencias}

1. Young P. La apendicitis y su historia. Revista médica de Chile [en línea]. mayo de 2014 [citado 4 de noviembre de 2016];142(5):667-72. Disponible

http://www.scielo.cl/scielo.php?script=sci abstract\&pid=S003498872014000500018\&lng=es\&nrm=iso\&tlng=es

2. Serrano Serrano TC. Valoración de la escala de Alvarado como herramienta diagnóstica para apendicitis aguda comparado con el resultado histopatológico en pacientes atendidos por el servicio de cirugía general en el Hospital General IESS Ambato en el período junio 2015-Noviembre 2015 [en línea] [B.S. thesis]. 2016 [citado 27 de octubre de 2016]. Disponible en: http://186.3.45.37/handle/123456789/5154

3. Memon AA, Vohra LM, Khaliq T, Lehri A. Diagnostic accuracy of Alvarado score in the diagnosis of acute appendicitis. Pak J Med Sci [en línea]. 2009 [citado 12 de abril de 2017];25(1):118-121. Disponible en: http://pjms.com.pk/issues/janmar09/article/article23.html

4. Seetahal SA, Bolorunduro OB, Sookdeo TC, Oyetunji TA, Greene WR, Frederick W, et al. Negative appendectomy: a 10-year review of a nationally representative sample. The American Journal of Surgery [en línea]. 2011[citado 23 de junio de 2016];201(4):433-7. Disponible en: http://www.sciencedirect.com/science/article/pii/S0002961011000286

5. Mendoza DJDV, Rodríguez DCG, Guerrero DMÁV. Evaluación prospectiva de la Escala de Alvarado en el diagnóstico de apendicitis aguda. Salamanca, España [en línea]. 2010 [citado 11 de enero de 
2017]; Disponible en: http://www.medigraphic.com/pdfs/cirgen/cg2010/cg101c.pdf

6. Cernadas C, M J. La OMS y su iniciativa «Alianza Mundial para la Seguridad del Paciente». Archivos argentinos de pediatría [en línea]. octubre de 2009 [citado 15 de febrero de 2017];107(5):385-6. Disponible http://www.scielo.org.ar/scielo.php?script=sci_abstract\&pid=S032500752009000500001\&lng=es\&nrm=iso\&tlng=es

7. González HMR, Yáñez IEP, Fajardo RCS, Hernández JEM, Chávez NAM. Prevalencia de apendicitis aguda en un centro de segundo nivel de atención. Cirujano General [en línea]. 2014 [citado 22 de enero de 2017];36(2):87-90. Disponible http://www.academia.edu/download/43787306/es-revista-cirujanogeneral-218-pdf-90355182-S300.pdf

8. Velázquez Mendoza J, Godínez Rodríguez C, Vázquez Guerrero M. Evaluación prospectiva de la Escala de Alvarado. Cir Gen. 2010;32:17-23.

9. Mason RJ, Moazzez A, Sohn H, Katkhouda N. Meta-Analysis of Randomized Trials Comparing Antibiotic Therapy with Appendectomy for Acute Uncomplicated (No Abscess or Phlegmon) Appendicitis. Surgical Infections [en línea]. 24 de febrero de 2012 [citado 13 de marzo de 2017];13(2):74-84. Disponible en: http://online.liebertpub.com/doi/abs/10.1089/sur.2011.058

10. Akinosoglou K, Kraniotis P, Thomopoulos K, Assimakopoulos SF. Epiploic appendagitis: a non-surgical cause of acute abdomen. Annals of gastroenterology: quarterly publication of the Hellenic Society of Gastroenterology [en línea]. 2015 [citado 25 de febrero de 2017];28(2):296. Disponible http://pubmedcentralcanada.ca/pmcc/articles/PMC4367230/

11. Larrañaga I, Mendía E, Die J, Lobo E. Abdomen agudo. Lobo Martínez $\mathrm{E}$, director Manual de urgencias quirúrgicas $2 \mathrm{a}$ ed Coordinación editorial: IM\&C. 2000;97-111.

12. Jiménez Aranda L, Tybos I, Fernández FL, Buforn Galiana A. Dolor abdominal en urgencias. MALAGA [en línea]. 2010 [citado 19 de abril de 2017]; Disponible en: http://www.medynet.com/usuarios/jraguilar/Manual\%20de\%20urgenci as $\% 20 y \% 20$ Emergencias/dolorabd.pdf
13. Wray CJ, Kao LS, Millas SG, Tsao K, Ko TC. Acute Appendicitis: Controversies in Diagnosis and Management. Current Problems in Surgery [en línea]. 2013 [citado 16 de febrero de 2017];50(2):54-86. Disponible

http://linkinghub.elsevier.com/retrieve/pii/S0011384012001967

14. Segev L, Keidar A, Schrier I, Rayman S, Wasserberg N, Sadot E. Acute Appendicitis in the Elderly in the Twenty-First Century. Journal of Gastrointestinal Surgery [en línea]. 2015[citado 26 de febrero de 2017];19(4):730-5. Disponible en: http://dx.doi.org/10.1007/s11605014-2716-9

15. Yu C-W, Juan L-I, Wu M-H, Shen C-J, Wu J-Y, Lee C-C. Systematic review and meta-analysis of the diagnostic accuracy of procalcitonin, C-reactive protein and white blood cell count for suspected acute appendicitis. British Journal of Surgery [en línea]. 2013[citado 21 de febrero de 2017];100(3):322-9. Disponible en: http://dx.doi.org/10.1002/bjs.9008

16. Flum DR. Acute Appendicitis - Appendectomy or the "Antibiotics First" Strategy. New England Journal of Medicine [en línea]. 2015[citado 20 de marzo de 2017];372(20):1937-43. Disponible en: http://www.nejm.org/doi/full/10.1056/NEJMcp1215006

17. Didier RA, Vajtai PL, Hopkins KL. Iterative reconstruction technique with reduced volume $\mathrm{CT}$ dose index: diagnostic accuracy in pediatric acute appendicitis. Pediatr Radiol [en línea]. 1 de febrero de 2015 [citado 13 de mayo de 2017];45(2):181-7. Disponible en: https://link.springer.com/article/10.1007/s00247-014-3109-7

18. Yokoe M, Takada T, Strasberg SM, Solomkin JS, Mayumi T, Gomi H, et al. TG13 diagnostic criteria and severity grading of acute cholecystitis (with videos). Journal of Hepato-Biliary-Pancreatic Sciences [en línea]. enero de 2013 [citado 21 de abril de 2017];20(1):35-46. Disponible http://doi.wiley.com/10.1007/s00534-012-0568-9

19. Kirkil C, Ilhan YS, Aygen E, Bulbuller N, Gulturk B, Coskun S. A retrospective analysis of the treatment results of 1557 patients with acute cholecystitis. J Pak Med Assoc [en línea]. 2015 [citado 15 de marzo de 2017];65:277-282. Disponible en: http://www.jpma.org.pk/PdfDownload/7287.pdf 